\title{
Dental implant training coming to Portsmouth
}

The University of Portsmouth Dental Academy will be launching a programme of continuing professional development (CPD) courses based around dental implant training for dentists and dental carve professionals (DCPs).

A series of short courses has been developed by Dr Peter Sanders (pictured), with the aim of allowing dentists and DCPs to build a strong foundation of knowledge and understanding of dental implants, focusing on practical skills in areas including impression taking, the fitting of implant restorations and general implant care in practice.

The introductory courses aim to provide dentists with the confidence, skills and knowledge that will allow their patients to access dental implants. Course participants will learn when implants should be offered as a treatment alternative and how to engage in providing simple implant treatments with the support of a suitable surgical partner/mentor, allowing implants to become a part of their scope of practice.

Dr Sanders explained: 'I've been involved in the FGDP implant diploma course

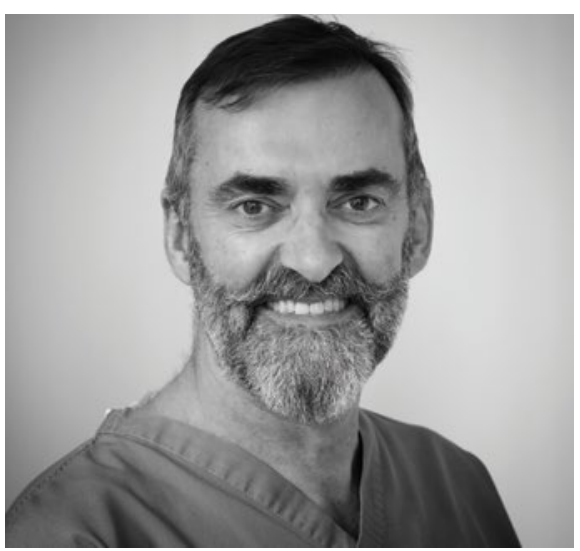

Dr Peter Sanders

for 15 years and still consider it to be the benchmark implant training programme. However, the one disappointment seen with many courses is the low percentage of course participants who sit the final assessments. The reason is almost invariably, that implantology is not within the scope of their daily practice. Without patients on whom to practise their newly acquired skills, the skills, knowledge and confidence are lost and their involvement in the course is therefore reduced to an academic exercise.
'What I have developed and now implemented within the Dental Academy at the University of Portsmouth is an inexpensive modular introductory programme to help colleagues start introducing implantology to their patients. With an experienced surgical partner and suitable mentor, implantology can benefit participants' patients and their practice. When implants become a regular feature of practice life, then advanced training becomes a sound clinical and financial investment. I'm very excited to be able to develop this series of CPD courses at the Dental Academy.

Dr Sanders has previously been Programme Director, Cohort Course Director and Examiner on the implantology course at the Royal College of Surgeons. He is Chief Examiner for the implantology MSc at King Juan Carlos University of Madrid and President of the Implant Diploma Alumni. He regularly speaks at key professional events at an international level, is a Zimmer Biomet Global Trainer and Past-President of the British Society for Advanced Dentistry (BSAD).

To view details of the programme visit www.port.ac.uk/dentalcpd.

\section{New research on dental X-ray risks}

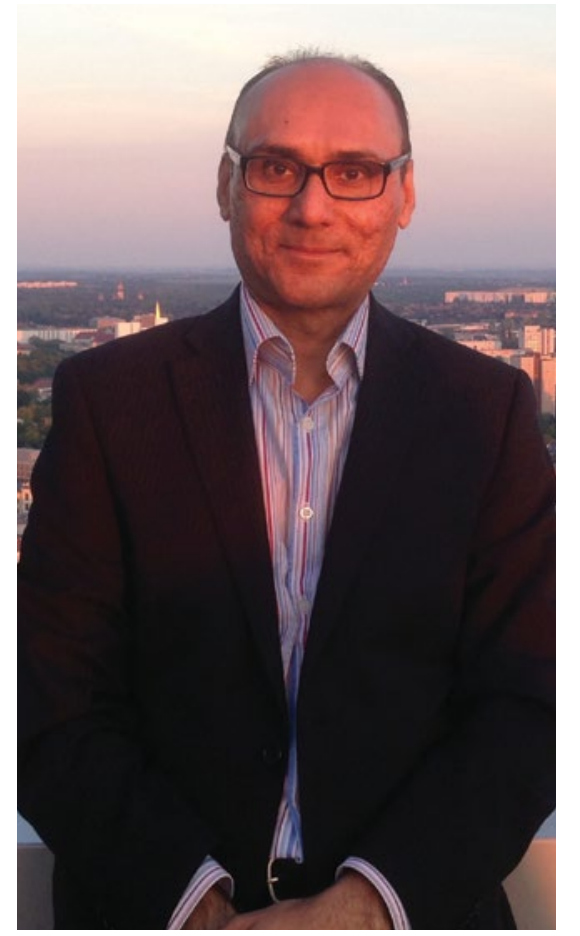

Professor Anjum Memon
Repeated exposure to dental X-rays may increase the risk of thyroid cancer and tumours in tissue covering the brain and spinal cord, according to new research. ${ }^{1}$

About 3,500 new cases of thyroid cancer and 1,850 'meningiomas' (tumours which are mostly benign and grow slowly) are diagnosed each year in the UK and researchers have discovered an increase in both diseases in many countries in the past 30 years.

Professor Anjum Memon, Chair in Epidemiology and Public Health Medicine at Brighton and Sussex Medical School, said much of the increase in thyroid cancers is 'probably due to increased surveillance, screening and over-diagnosis (ie detection of a cancer that would not ultimately cause symptoms), but we believe other causes need investigation'.

Of the thyroid gland, situated in the neck and the meninges which cover the brain and spinal cord, he said: 'These organs will be exposed to radiation from dental X-rays.
Both organs are highly radiosensitive, particularly in childhood and adolescence. Dental radiography, a source of low-dose diagnostic radiation, is often overlooked as a potential hazard to these organs. He said more research was needed to further test the hypothesis.

Professor Memon said the results of their research 'should be treated with caution because these studies did not include individual organ doses and ages at exposure, and are subject to recall bias and other limitations'. The researchers said that their synthesis provides good evidence to warrant more research based on dental $\mathrm{X}$-rays records and patient follow-up to test the hypothesis further.

\section{Reference}

1. Memon A, Rogers I, Paudyal P, Sundin J. Dental $x$-rays and the risk of thyroid cancer and meningioma: a systematic review and meta-analysis of current epidemiological evidence. Thyroid 2019; DOI: 10.1089/ thy.2019.0105 [Epub ahead of print]. 